\title{
Advances in understanding of the primary reactions of protochlorophyll(ide) photoreduction in cells and model systems
}

\author{
Olga B. Belyaeva, Felix F. Litvin
}

Faculty of Biology, Lomonosov Moscow State University, Moscow, Russia; belyaeva0104@gmail.com

Received 6 November 2010; revised 15 November; accepted 19 November 2010.

\begin{abstract}
The key step in chlorophyll biosynthesis is photoreduction of its immediate precursor, protochlorophyllide. This reaction is catalyzed by a photoenzyme, protochlorophyllide oxidoreductase (POR) and consists in the attachment of two hydrogen atoms in positions $\mathrm{C} 17$ and $\mathrm{C} 18$ of the tetrapyrrole molecule of protochlorophyllide; the double bond is replaced with the single bond. Two hydrogen donors involved in protochlorophyllide photoreduction are NADPH $[1,2]$ and a conserved tyrosine residue Tyr193 of the photoenzyme POR [3]. The structure of active pigment-enzyme complex (Pchlide-POR-NADPH) ensures a favorable steric conditions for the transfer of hydride ion and proton. This review does not examine the ternary complex structure, but concentrates upon the mechanisms of primary photophysical and photochemical reactions during formation of chlorophyllide from protochlorophyllide in living objects (etiolated leaves and leaf homogenates) and model systems.
\end{abstract}

Keywords: Protochlorophyllide; Chlorophyllide; Photoreduction; Fluorescence; Spectroscopy

\section{PRIMARY REACTIONS OF PROTOCHLOROPHYLLIDE PHOTOREDUCTION IN PLANT LEAVES AND ISOLATED PIGMENT-PROTEIN COMPLEXES}

\subsection{Labile Intermediates Stabilized at Low Temperatures}

Analysis of protochlorophyllide photoreduction in vivo at very low temperatures, at which biochemical temperature-dependent steps are inhibited, helped to clarify the mechanism of this reaction. Rubin et al. [4] reported that the fluorescence lifetime in etiolated leaves was found to decreased upon elevation of sample temperature from $77 \mathrm{~K}$ to $193 \mathrm{~K}$. The further temperature increase in darkness led to the appearance of fluorescence bands characteristic of chlorophyll. The authors supposed that the absorption of fluorescence-exciting light at low temperatures converted the protochlorophyllide molecule into the intermediary state, which was transformed to chlorophyllide during subsequent dark reaction allowed to proceed at higher temperature. This assumption was confirmed in later studies $[5,6]$. By illuminating leaves at $153 \mathrm{~K}$, Raskin [7] successfully stabilized the intermediate product of photochemical reaction and could determine the spectral position of its absorption band (near $690 \mathrm{~nm}$ ). Later, a similar position of absorption maximum for the primary intermediate was detected at room temperature $[8,9]$. The nonfluorescent intermediate stabilized at low temperatures (intermediate X690) was investigated in many research groups [10-19]. It was found that the primary photoreaction could proceed even at the temperature of liquid helium $(4.2 \mathrm{~K})$ [20]. This finding revealed the elementary photophysical nature of the process accompanied by quenching of protochlorophyllide fluorescence.

In the dark the nonfluorescent intermediate X690 transforms into chlorophyllide upon the increase in temperature:

$$
\text { Pchlide 655/650 } \stackrel{\mathrm{h} v}{\longrightarrow} \text { X690 } \rightarrow \text { Chlide }
$$

\subsection{Occurrence of SeveralShort-Lived NonFluorescent Intermediates}

Studies of primary events in protochlorophyllide photoreduction at very low temperatures led to the conclusion that formation of intermediate $\mathbf{X 6 9 0}$ is preceded by even earlier photochemical reactions. Evidence to support this notion came from comparison of spectral changes induced by illumination of etiolated leaves at 
various temperatures [15,20-22].

Fluorescence and absorption spectroscopy studies have shown the reversibility of the primary reaction that converts protochlorophyllide into the nonfluorescent intermediate [16]. Calculations of the rate constants and quantum yields of the forward and backward photoreactions under photostationary equilibrium showed that the low quantum efficiency of the whole process could not be due to photoreversibility of $\mathbf{X 6 9 0}$-producing reaction because the quantum yield of the backward process is significantly lower (by a factor of 20). Since the intermediate X690 is stable in darkness, the authors assumed that $\mathbf{X 6 9 0}$ formation is preceded by one additional intermediary step that comprises a fast backward reaction responsible for the lowered yield of the whole process. The putative intermediate was designated by the symbol R (reversible):

$$
\text { Pchlide 655/650 } \stackrel{\mathrm{h} v}{\longrightarrow} \mathbf{R} \rightarrow \mathbf{X 6 9 0} \rightarrow \text { Chlide }
$$

The above experimental data gave clue to explanation why the quantum yield of the reaction at low temperature is lower than the quantum yield of the process at room temperature when it is close to 0.5 . The rate constants of forward processes increase faster upon raising the temperature than those of backward reactions, which elevates the total yield of chlorophyllide formation.

Analysis of spectral changes led to the suggestion that the absorption spectrum of the primary intermediate $\mathrm{R}$, whose production dominates at $77 \mathrm{~K}$, is close or even identical to the absorption spectrum of the protochlorophyllide active form: the primary action of light on etiolated leaves, evident in quenching of protochlorophyllide fluorescence, occurs under conditions when X690 is hardly discernible in absorption spectra [21].

Further evidence that the chain of protochlorophyllide transformations includes the short-lived intermediate with the absorption band similar to that of protochlorophyllide active form emerged from the time-resolved spectroscopy studies performed at room temperature $[19,23]$.

We examined the early stages of protochlorophyllide photoreduction by comparing low-temperature $(77 \mathrm{~K})$ optical spectra (absorption and fluorescence) and ESR spectra of whole etiolated leaves [21]. After illumination of leaves at $77 \mathrm{~K}$, when the production of nonfluorescent intermediate $\mathbf{R}$ is predominant, a structureless singlet ESR signal was observed with a bandwidth of $1.1 \mathrm{mT}$ and a g-factor of 2.0021 characteristic of the free electron. As the sample temperature was raised gradually to $200 \mathrm{~K}$, the ESR signal amplitude increased in parallel with narrowing of the signal width. Such a pattern of ESR signal changes apparently corresponds to the transformation of the primary intermediate $\mathbf{R}$ into the inter- mediate X690. Upon further increase in temperature above $200 \mathrm{~K}$, the ESR signal amplitude dropped abruptly, reaching its initial dark level at the temperature of about $250 \mathrm{~K}$. These changes occurred synchronously with the appearance of fluorescence spectral bands assigned to primary forms of chlorophyllide.

Studies of the primary steps of protochlorophyllide photoreduction in etiolated leaves by means of differential and derivative absorption spectroscopy [24] provided evidence for even higher complexity of the photoprocess. The intermediate $\mathbf{X 6 9 0}$ was found to exist in two forms distinguished by absorption maxima at 697 and $688 \mathrm{~nm}$ and by different rates of their production. These intermediates were designated as R697 and R688. After short-term illumination of leaves at $77 \mathrm{~K}$, the subtracted "light minus dark" spectrum contained a weak band with the maximum at $697 \mathrm{~nm}$ (intermediate R697) while the amplitude of the protochlorophyllide band $(650 \mathrm{~nm})$ remained unchanged. During prolonged illumination, a band at $688 \mathrm{~nm}$ appeared in the subtracted spectrum in addition to the former band at $697 \mathrm{~nm}$. At the same time, the decrease in the absorption band of active protochlorophyllide form at $650 \mathrm{~nm}$ was observed, indicating that the protochlorophyllide molecule underwent at this stage more profound changes than at the stage of intermediate $\mathbf{R 6 9 7}$ formation, when the absorption at $650 \mathrm{~nm}$ remained almost unchanged. According to ESR spectra, both the intermediates R697 and R688 exhibit paramagnetic properties. The dark stage of chlorophyllide formation during temperature increase was only observed after accumulation of intermediate $\mathbf{R 6 9 7}$ in combination with $\mathbf{R 6 8 8}$. A proposal was put forward that these two intermediates are the products of two parallel photoreactions proceeding at different rates; the temperature increase promotes interaction of their chromophores, giving rise to chlorophyllide molecule through disproportionation of free radicals. The mechanism of chlorophyllide formation by means of free radical disproportionation was earlier proposed by Losev and Lyalkova [14].

Summing up this section, we conclude that photoreduction of protochlorophyllide in vivo comprises at least two elementary reactions which can be detected by spectral methods at very low temperatures required for stabilization of nonfluorescent intermediates arising as the products of these photoreactions:

$$
\begin{aligned}
& \text { Pchlide } 655 / 650 \stackrel{\mathrm{h} v}{\longrightarrow} \mathrm{R}-/ 650 \rightarrow \mathrm{X} 690(\mathrm{R} 697+\mathrm{R} 688) \\
& \rightarrow \text { Chlide }
\end{aligned}
$$

Since the intensity of the absorption band for the intermediate R697 is very low (it is only observed in differential spectra), a possibility cannot be ruled out that this primary product is identical to the intermediate R-/650. 


\section{RAPID STAGES OF PROTOCHLOROPHYLLIDE PHOTOREDUCTION IN VIVO AT PHYSIOLOGICAL TEMPERATURES}

An alternative approach to detection of labile shortlived intermediates involved in protochlorophyllide photoreduction was based on time-resolved spectroscopy employed in studies of chlorophyll photobiosynthesis in etiolated leaves. The application of these methods allowed researchers to detect short-lived intermediates at physiological temperatures.

The kinetic studies of protochlorophyllide fluorescence and fluorescence changes of chlorophyllide produced in etiolated leaves after illumination by short $(0.3$ ms) flashes at room temperature revealed that the chlorophyllide fluorescence intensity increased toward maximum in $400 \mathrm{~ms}$, whereas the protochlorophyllide fluorescence dropped to the minimum within less than 8 $\mathrm{ms}$ [25]. The discrepancy between stabilization times of chlorophyllide and protochlorophyllide fluorescence intensities indicated the production of a nonfluorescent intermediate, which was converted during the dark step to the fluorescent form of chlorophyllide. The application of even shorter light pulses (20 ns) and a sensitive technique for scanning the absorption spectra of etiolated leaf homogenates at room temperature [8] revealed the appearance within $0.5 \mu$ s after the flash of an unstable product with the absorption maximum around 695 $\mathrm{nm}$, which transformed within few microseconds into chlorophyllide with the absorption maximum near 675 $\mathrm{nm}$. Similar results were also obtained in studies of rapid changes in absorption spectra of etiolated leaves and isolated pigment-protein complexes [9]. Inoue et al. discovered the primary short-lived intermediate $(0.2 \mu \mathrm{s})$ with the absorption maximum at $690 \mathrm{~nm}$.

Iwai et al. [23] investigated the primary steps of protochlorophyllide photoreduction using active pigmentprotein complexes isolated from etiolated leaves and the nanosecond- and picosecond absorption spectroscopy. At physiological temperature on the nanosecond and microsecond time scale, they observed the appearance of three intermediates $\left(\mathrm{X}_{1}, \mathrm{X}_{2}, \mathrm{X}_{3}\right)$ with characteristic time constants of $2 \mathrm{~ns}, 35-250 \mathrm{~ns}$, and 1-2 $\mu \mathrm{s}$, which was followed by chlorophyllide formation $(12 \mu \mathrm{s})$. The absorption peak of $X_{1}$ is close to the absorption maximum of the initial protochlorophyllide $(\geq 640 \mathrm{~nm})$. The intermediates $\mathrm{X}_{2}$ and $\mathrm{X}_{3}$ are characterized by the absorption maxima at 688 and $684 \mathrm{~nm}$, respectively. Using flash photolysis (a 30-ps flash), these authors showed the existence of even earlier intermediate, termed $\mathrm{X}_{0}$, which appeared at room temperature within the period of $\leq 50$ ps from the protochlorophyllide excitation and had the lifetime of about 1-2 ns (compatible with the results from other group [26]). The position of absorption maximum of the primary product $\mathrm{X}_{0}$ was almost coincident with the band position of the initial protochlorophyllide. The time constant of $\mathrm{X}_{0}$ formation corresponded to the relaxation time of the protochlorophyll molecule from the Franck-Condon state $\left(\mathrm{S}_{1} *\right)$ to the equilibrium state $\left(S_{1}\right)$; hence the $X_{0}$ entity is not an intermediate in a chemical sense. The following scheme was proposed for the primary events of protochlorophyllide photoreduction in active pigment-protein complexes:

$$
\begin{aligned}
& \text { Pchlde }\left(\mathrm{S}_{0}\right) \stackrel{\mathrm{h} v}{\longrightarrow} \text { Pchlde }\left(\mathrm{S}_{1}^{*}\right) \stackrel{\leq 50 \mathrm{ps}}{\longrightarrow} \text { Pchlde }\left(\mathrm{S}_{1}\right) \\
& \stackrel{1-2 \mathrm{~ns}}{\longrightarrow} \mathrm{x}_{1} \stackrel{35-250 \mathrm{~ns}}{\longrightarrow} \mathrm{x}_{2} \stackrel{1-2 \mu \mathrm{s}}{\longrightarrow} \mathrm{x}_{3} \stackrel{12 \mu \mathrm{s}}{\longrightarrow} \text { Chlde }
\end{aligned}
$$

The authors supposed that the intermediate $X_{3}$ corresponds to the nonfluorescent intermediate $\mathbf{X 6 9 0}$ observed in etiolated leaves after illumination at low temperatures. However, according to other authors [27,28], the intermediate $\mathbf{X 6 9 0}$ is more likely to correspond with the intermediate $\mathrm{X}_{2}$ observed by Iwai et al. This view is based on the absorption spectrum of this intermediate (maximum at $688 \mathrm{~nm}$ ) and on its rise time (35-250 ns), which is comparable to the formation time of intermediate X690 determined by other researchers [8,29]. The intermediate $X_{1}[23]$ is likely identical to the intermediate $\mathbf{R}$ discovered in our work $[16,24]$, as evidenced from similarity of its absorption spectrum to the absorption spectrum of the initial protochlorophyllide. The intermediate $\mathrm{X}_{3}$ can be compared with one of the primary forms of chlorophyllide arising in etiolated leaves after preillumination at $77 \mathrm{~K}$ and subsequent return to higher temperature.

Thus, the comparative analysis of the research results concerning intermediate stages of protochlorophyllide photoreduction in vivo at physiological and low temperatures leads to the conclusion that this process comprises several intermediary products, including two or three short-lived intermediates characterized by strong quenching of protochlorophyllide fluorescence.

\section{PRIMARY FLUORESCENT FORMS OF CHLOROPHYLLIDE}

The nonfluorescent intermediate $\mathbf{X 6 9 0}$, stabilized effectively at low temperatures, transforms into chlorophyllide in dark reactions after raising the temperature of preilluminated sample. When white light was used for illumination of etiolated leaves at $77 \mathrm{~K}$, the return to higher temperature was followed by almost simultaneous formation of two primary forms of chlorophyllide with fluorescence maxima at 684 and $695 \mathrm{~nm}$ and the respective absorption maxima at 676 and $684 \mathrm{~nm}$ [15]. Thereafter, the long-wavelength form Chlide695/684 was converted in the dark reaction to a short-wavelength form Chlide684/676. This pigment is presumably the 
chlorophyllide form, which is a starting structure in the biosynthetic pathway giving rise to the reaction center pigments of photosystem II and to the pigments of light-harvesting complex (reviewed in [30]). The proportion of 695 and $684 \mathrm{~nm}$ fluorescence bands arising after illumination at low temperature depended on spectral quality of actinic light. When blue light (with the peak intensity at $470 \mathrm{~nm}$ ) was used for illumination, the increase in temperature resulted in formation of the only band at $695 \mathrm{~nm}$, which shifted gradually to $684 \mathrm{~nm}$. When the leaves were preilluminated with red light (with wavelengths above $600 \mathrm{~nm}$ ), the temperature rise was followed by the appearance in the spectrum of only one short-wavelength maximum at $684 \mathrm{~nm}$. In etioplast preparations only one short-wavelength chlorophyllide form was produced. These data implied that etiolated leaves contain two kinds of active protochlorophyllide-protein complexes exhibiting nearly identical absorption and fluorescence bands in the red spectral region [15]. Both forms are converted to chlorophyllide through the stage of formation of nonfluorescent intermediate. One of these precursors is able to transform immediately into a short-wavelength chlorophyllide form Chlide684/676. The reaction scheme was presented as follows:

$$
\begin{aligned}
& \text { Pchld 655/650 } \stackrel{\mathrm{h} v}{\longrightarrow} \mathrm{R} \rightarrow X 690 \rightarrow \text { Chld 695/684 } \\
& \text { Pchld 655/650 } \stackrel{\mathrm{h} v}{\longrightarrow} \mathrm{R} \rightarrow X 690 \rightarrow \text { Chld 695/684 } \rightarrow
\end{aligned}
$$

Later studies demonstrated the existence in young etiolated leaves of an additional active protochlorophyllide form Pchlide653/648 [30,31] possessing a blueshifted absorption band in the Soret region $(440 \mathrm{~nm})$, as compared to the band position for the main active form Pchlide655/650 (450 nm). Pchlide653/648 is produced under the action of red light from the minor long-wavelength form of the precursor Pchlide686/676 [31]. Furthermore, we cannot exclude the possibility that one of the two reactions consists in the conversion of a short-wavelength active precursor Pchlide 643/637, whose fluorescence is quenched owing to highly effective energy transfer toward the main active form.

Belyaeva and Sundqvist [32] revealed the formation of four primary fluorescent pigment forms. When etiolated leaves preilluminated at $77 \mathrm{~K}$ were heated to $190 \mathrm{~K}$, a broad structured band appeared in the red region of their fluorescence spectrum. The decomposition of the spectra to the Gaussian components proved that this band consists of four components with maxima at 683, 690, 696, and $706 \mathrm{~nm}$. Apparently, the formation of several primary labile chlorophyllide forms points to the early onset for differentiation pathways involved in formation of functionally different native pigment forms.

\section{OBSERVATION OF ELEMENTARY REACTIONS OF PROTOCHLOROPHYLL(IDE) PHOTOREDUCTION IN SOLUTIONS}

Studies of protochlorophyll reduction in solutions provided another approach to elucidating the mechanism of primary steps in chlorophyll biosynthesis from its precursor and the role of biological structures in this process. This line of research enables identification of spectral changes related to the conversions of the chromophore molecule per se.

In order to gain deeper insight into the earliest stages of protochlorophyll(ide) reduction, based on the previous studies with whole leaves, we examined this process in solutions and pigment films in cooperation with Bystrova and Timofeev [21,34]. The experimental conditions were maximally similar to those proved successful for detecting the primary stages of protochlorophyllide reduction in plant leaves (high intensity short-term illumination at $77 \mathrm{~K}$ ). We found out that the primary photophysical stages of protochlorophyllide reduction could occur in solutions and films in the absence of reducing agents. In this case the role of hydrogen donor belongs apparently to the solvent (ethanol, ethyl ether, and pyridine).

When diluted solutions of protochlorophyll and protochlorophyllide in ethyl ether $\left(10^{-6} \mathrm{M}\right)$ were illuminated at $77 \mathrm{~K}$, the fluorescence at $626 \mathrm{~nm}$ attributed to the monomeric pigment form was quenched, but this quenching was fully reversed upon the subsequent elevation of temperature to $273 \mathrm{~K}$. No changes in the absorption spectrum were detected [34]. The quenching of protochlorophyll fluorescence occurred synchronously with the appearance in the ESR spectrum of the singlet signal with the g-factor of 2.0013 and the singlet band width of $1.2 \mathrm{mT}$; this signal disappeared rapidly upon the increase in temperature in parallel with the buildup of protochlorophyllide fluorescence [21]. The addition of reducing agents resulted in a severalfold acceleration of fluorescence quenching. The comparison with the photoreduction of chlorophyll precursor in whole etiolated leaves suggests that the model systems with monomeric protochlorophyllide could mimick the earliest stage of the pigment photoreduction producing the paramagnetic intermediate $\mathbf{R}$.

Spectral features of concentrated protochlorophyll solutions in ethanol $\left(10^{-4} \mathrm{M}\right)$ imitated quite well the absorption and fluorescence spectra of three main forms of chlorophyll precursor: the spectra of these solutions contained absorption bands at 625,635 and $645-7 \mathrm{~nm}$ and fluorescence bands at 627, 637, and 651-2 nm. Measurements of circular dichroism spectra for protochlorophyll in model systems $[45,46]$ indicate that the spectral band at 651-652 nm, in the farthest long-wave- 
length position, is determined by the formation of pigment dimers. Illumination of concentrated ethanolic solution at $77 \mathrm{~K}$ led to quenching of all fluorescence bands $[21,34]$. Fluorescence quenching developed synchronously with the appearance in low-temperature absorption spectrum of a weak maximum around $690 \mathrm{~nm}$, which is close to the position of absorption peak for nonfluorescent intermediate X690 discovered in plant leaves. In the ESR spectrum, a singlet signal with the g-factor of 2.0015 and bandwidth of $1.1 \mathrm{mT}$ appeared upon illumination (the signal parameters were almost identical to those induced by illumination in etiolated leaves). Hence, the aggregated protochlorophyll in model systems, unlike the monomeric pigment, undergoes more profound transformation including the formation of nonfluorescent intermediate X690. Apparently, this stage of the process involves the aggregated long-wavelength pigment form.

Thus, elementary reactions of pigment photoreduction occurring in the plant cell can be reproduced in protochlorophyll solutions as a model system

\section{ELEMENTARY REACTIONS OF PROTOCHLOROPHYLLIDE PHOTOREDUCTION IN RECONSTITUTED TERNARY COMPLEXES}

In the last few years a growing number of researchers turned to studying protochlorophyllide photoreduction with the use of artificial ternary complexes (consisting of protochlorophyllide, NADPH, and photoenzyme protochlorophyllide oxidoreductase), because the properties of such complexes are most close to those of living systems. The main active protochlorophyllide form in such systems is commonly represented by the form whose spectral properties are similar to those of protochlorophyllide active form in vivo, Pchlide 643/637 [17,18,38]. However, the reconstituted ternary complexes obtained in our joint studies with Griffiths were characterized by spectra with fluorescence bands at 651 and $708 \mathrm{~nm}$. The position of the first band coincides with the maximum of protochlorophyllide fluorescence in whole cells, while the second band was ascribed to a small number of large aggregates serving as a sink for effective energy transfer [37].

Similarly to observations with whole etiolated leaves, the protochlorophyllide photoreduction in artificial ternary complexes was found to produce unstable nonfluorescent intermediate as a primary product arising after illumination of samples at low temperatures $[17,18,37$, 38]. The absorption band of this product had a peak at $696 \mathrm{~nm}[18,38]$. The quenching of fluorescence was paralleled by the appearance in the ESR spectrum of a singlet signal with the g-factor characteristic of the free electron [37]. Upon the increase in temperature, the nonfluorescent intermediate was converted into chlorophyllide. The production of chlorophyllide after the temperature rise was evident from the appearance of characteristic bands in spectra of methanolic extracts [37].

Heyes et al. [19] examined the protochlorophyllide photoreduction in reconstituted ternary complexes at room temperature using femtosecond spectroscopy (50-fs laser flash with a wavelength of $475 \mathrm{~nm}$ ). Under these conditions, a small increase in absorption of the initial protochlorophyllide (maximum at $642 \mathrm{~nm}$ ) was observed in the time range between 3 and 400 ps after the flash, which was accompanied by a slight shift of the peak position to the short-wavelength spectral region. At the same time, a weak maximum around $677 \mathrm{~nm}$ emerged. These results are consistent with investigations of the living systems: in both cases the primary reaction was characterized by the lack of protochlorophyllide bleaching (in parallel with effective fluorescence quenching) $[23,24]$.

Thus, protochlorophyllide photoreduction in reconstituted ternary complexes is implemented in at least two stages: the light-induced formation of a short-lived nonfluorescent intermediate characterized by weak ESR signal with the free electron g-factor, followed by the dark transformation of this intermediate into chlorophyllide. The pattern of spectral changes is close to that observed in etiolated leaves.

\section{POSSIBLE MECHANISMS IN PHOTOREDUCTION OF PROTOCHLOROPHYLLIDE MOLECULE}

In our early pubication [15] we proposed that the cell primary reactions of protochlorophyllide photoreduction, characterized by the production of paramagnetic intermediates, proceed via consecutive transfer of two electrons and two protons toward the semi-isolated double bond $\mathrm{C} 17=\mathrm{C} 18$ in the protochlorophyllide molecule, resulting in the appearance of intermediary semireduced form, in essential similarity to a stepwise reduction of the double bond described for the photoreaction of porphyrin reduction in solutions. When the role of NADPH as a hydrogen donor was established [1,2], an assumption was put forward that the sequential transfer of two electrons is unlikely because of the high energy of nicotinamide radicals. Since the photoenzyme POR is structurally analogous to short-chain alcohol dehydrogenases, it seemed more probable that the protochlorophyllide photoreduction involves the transfer of hydride ion from NADPH in essential similarity to the mechanism ensuring substrate reduction by alcohol dehydro- 
genases. When NMR spectroscopy was applied for analysis of protochlorophyllide photoreduction in reaction mixtures containing protochlorophyllide, etioplast membranes, and NADPH isomers labeled with radioactive hydrogen $\left({ }^{3} \mathrm{H}\right)$, the results revealed that the hydride ion delivered from the $4 \mathrm{~S}$ position of nicotinamide moiety of NADPH is attached to the $\mathrm{C}_{17}$ position of protochlorophyllide, thus performing trans-reduction of the pigment molecule $[39,40]$. The notion that hydride ion and proton transfer are time-separated events during photoreduction of protochlorophyllide molecule is supported by the existence of several short-lived intermediates. The second proton is apparently donated by POR from the conserved amino acid residue Tyr-193 located in the vicinity of $\mathrm{C} 17=\mathrm{C} 18$ bond $[3,41,42]$. According to the homology model of POR enzyme [43], the D-ring of protochlorophyllide molecule is fixed against NADPH and the tyrosine residue, which facilitates the transfer of hydride ion and proton. The conserved residues Lys197 and Cys226 play an important role in configuring optimal structure of the ternary complex, thus enabling adequate steric requirements for the reaction of protochlorophyllide photoreduction [3,41-44]. The proton of tyrosine phenol group arrives at $\mathrm{C}_{18}$ atom of protochlorophyllide molecule. In reconstituted ternary complexes comprising mutant POR where Tyr-193 was replaced with Phe, the reduction of protochlorophyllide ceased at the stage of intermediary photoproduct, while the second (light-independent) stage of the reaction was inactivated [41]. The results indicate that the transfer of hydride ion occurs prior to the proton transfer.

Paramagnetism of electron donors or acceptors, manifested in the light-induced ESR signal is one of the most convincing evidence that the charge-transfer complex (CT complex) is formed in the donor-acceptor system. The formation of charge-transfer complex is accompanied by the appearance of new absorption band on the condition that the oxidation-reduction potentials of interacting partners are sufficient for completing the electron transfer from one to the other molecule within the complex. When the CT complex formation is due to partial charge transfer, the fluorescence quenching is observed without the appearance of new bands in the absorption spectrum. The possibility that photoreduction of protochlorophyllide in vivo proceeds through the stage of charge-transfer complex was considered in a series of studies $[7,14,22,27,28]$. Judging from spectral characteristics of two primary intermediates in vivo (termed as $\mathbf{R}$ and X690 in our publications $[16,21,22]$ or $X_{1}$ and $X_{2}$ according to [23]), one may suppose that photoinduced formation of these intermediates corresponds to the formation of complexes with partial charge transfer $\left[D^{\delta+}\right.$ $\left.\mathrm{A}^{\delta}\right]$ and with complete charge transfer $\left[\mathrm{D}^{+} \mathrm{A}^{-}\right][22,25,28]$ :

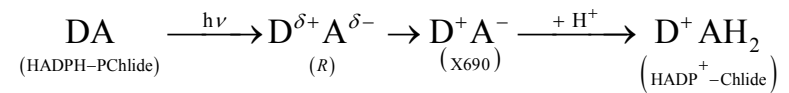

Raskin and Schwartz considering the investigation [23] proposed that a comparatively long lifetime of the intermediate $\mathrm{X}_{1}(35-250 \mathrm{~ns})$ indicates the existence at this stage of two intermediates: the singlet exciton complex (exiplex) and the triplet exiplex of protochlorophyllide and the hydrogen donor. Examination of lowtemperature phosphorescence spectra of whole etiolated leaves also supports the viewpoint that illumination of the leaves at $77 \mathrm{~K}$ (the stage of formation of nonfluorescent intermediate) produces not only singlet exiplexes but also triplet exiplexes of protochlorophyllide and the hydrogen donor, because fluorescence quenching of active protochlorophyllide form under these conditions was concomitant with quenching of its phosphorescence [45]. In our opinion, further experiments are needed to answer the question about the role of triplet state of the protochlorophyllide molecule in its photoreduction.

Heyes et al. [38] attempted to elucidate the photophysical mechanism of protochlorophyllide reduction in the ternary complexes by comparative study of this reaction at $180 \mathrm{~K}$ using absorption spectroscopy, ESR spectroscopy, ENDOR spectroscopy, and Stark spectroscopy. In illuminated samples the authors detected ESR spectra suggesting the emergence of two paramagnetic products. However, quantitative estimates based on ESR spectra indicated that essentially complete transformation of the active protochlorophyllide form (as estimated from changes in absorption spectra) resulted in only $5 \%$ output of the pigment free radicals. The appearance of nonfluorescent intermediate with the absorption band at 696 $\mathrm{nm}$ corresponded to the broadband Stark effect characteristic of charge transfer steps. The results obtained with Stark spectroscopy provided evidence for the existence of two constituents of the nonfluorescent intermediate. This enabled the authors to suggest that the primary stage of protochlorophyllide photoreduction is associated with the formation of charge-transfer complex. The temperature dependences of the intermediate formation and NADPH oxidation were identical. Therefore, the authors supposed that the formation of nonfluorescent intermediate involves the transfer of hydride ion for the creation of charge-transfer complex. The authors assumed that the photon absorption by the protochlorophyllide molecule leads to the temporary charge separation along the double $\mathrm{C} 17=\mathrm{C} 18$ bond, which facilitates the ultrafast transfer of hydride ion from NADPH to the C17 atom of protochlorophyllide [46, 38]. The resulting CT complex promotes the proton transfer toward the $\mathrm{C} 18$ atom in the subsequent dark reaction.

Heyes et al. [47] studied temperature dependences and 
isotopic effects of rate constants for two sequential reactions - the hydride anion and proton transfer steps - and analyzed data in the form of Eyring plots to obtain thermodynamic parameters for each step. The experimental results and calculations based on the density functional theory were consistent with a proton-tunneling, which requires fast (sub-picosecond) promoting motions coupled to the reaction coordinate.

Comparative studies of molecular pathways for protochlorophyllide photoreduction in vivo in various model systems allows the conclusion that the primary stages characterized by fluorescence quenching and formation of paramagnetic products are identical for whole etiolated leaves and all model systems examined, including the most simple ones such as low-concentrated pigment solutions. Nevertheless, some factors suggest that the mechanism of protochlorophyllide photoreduction in vivo is somewhat different from the mechanism of the reaction in model systems. The configuration of active complex in vivo seems to be more sophisticated than the ternary complex reconstituted in vitro, comprising three main components, i.e., Pchlide, NADPH, and POR. Specifically, this is indicated by different spectral characteristics of artificial and natural active complexes. Etiolated leaves contain several spectrally different active forms of protochlorophyllide. The major forms include Pchlide 643/639, Pchlide655/650, and the form Pchlide653/648, which is accumulated and metabolically converted in juvenile leaves. There are also several minor longwavelength forms participating in the process of protochlorophyllide photoreduction [48]. The reconstituted ternary active complexes usually comprise one or two protochlorophyllide forms: Pchlide633/630 (the form active only at room temperature) and Pchlide644/641, which remains photoactive also at low temperatures. The photoreduction of various protochlorophyllide forms in vivo and in vitro may involve different mechanisms. Evidence that protochlorophyllide photoreduction in vivo is accomplished via more intricate mechanism is evidenced by studies of this process in whole etiolated leaves at low temperature $[15,20]$, as well as by the results of time-resolved spectroscopy at physiological temperatures [21]; all these studies demonstrated the multistep nature of the reaction of protochlorophyllide photoreduction.

The presence of flavins in the active pigment-protein complex and their possible involvement in primary reactions of protochlorophyllide photoreduction was supposed in some studies [49-51]. Our investigations support this proposal, as we observed the drop of flavin fluorescence at $520 \mathrm{~nm}$ after illumination of leaves at 77 $\mathrm{K}$, which was reversed upon the return to higher temperature [51]. The oxidation-reduction reactions between nicotinamide and flavins are implemented by means of hydride ion transfer [52], while many flavin-containing enzymes participate in one-electron redox reactions. In plant leaves flavins can act at the intermediate stage of the reaction by acquiring hydride ion from NADPH and donating electron to the pigment molecule. In this case the reaction can include a stepwise transfer of two electrons and two protons and the formation at the first step of a semireduced pigment molecule with one attached electron (intermediary semireduced form). This mechanism is largely similar to that known for the reactions of porphyrin photoreduction in solution. We cannot rule out the existence of two mechanisms by which hydrogen atoms are transferred to protochlorophyllide from $\mathrm{NADPH}$ in whole etiolated leaves; i.e., the direct transfer of hydride ion from NADPH to $\mathrm{C} 17$ position in the protochlorophyllide molecule with the consequent attachment of proton and (or) flavin-mediated electron transfer from NADPH to the pigment. It is also possible that different mechanisms exist for the primary reactions of spectrally distinct active forms of protochlorophyllide.

Thus, we arrive at the conclusion that the mechanism of the primary elementary photophysical reactions during protochlorophyllide photoreduction is not yet completely elucidated. However, based on the results of numerous investigations, it can be stated that the reduction of active forms of the chlorophyll precursor is a multistep process comprising two or three short-lived intermediates characterized by the ESR singlet signal; the sequential conversions of these intermediates are ensured by structurally sophisticated native enzyme-pigment complex comprising protochlorophyllide, POR, and NADPH as its main constituents. Owing to specific structure of active ternary complex, favorable steric conditions are enabled for the reaction of protochlorophyllide photoreduction.

\section{REFERENCES}

[1] Griffiths, W.T. (1974) Source of reducing equivalent for the in vitro synthesis of chlorophyll from protochlorophyll. FEBS Letters, 46, 301-304.

doi:10.1016/0014-5793(74)80392-3

[2] Griffiths, W.T. (1975) Characterization of the terminal stages of chlorophyll(ide) synthesis in etioplast membrane preparations. Biochemistry Journal, 152, 623-635.

[3] Wilks, H.M. and Timko M.P. (1995) A light-dependent complexation system for analysis of NADPH: protochlorophyllide oxidoreductase identification and mutagenesis of two conserved residues that are essential for activity. Proceedings of the National Academy of the Sciences of the USA, 92, 724-728. doi:10.1073/pnas.92.3.724

[4] Rubin, A.B., Minchenkova, L.E., Krasnovsky, A.A. and Tumerman, L.A. (1962) Investigation of protochlorophyllide fluorescence lifetime during greening of etiolated leaves. Biofizika, 7, 571-577. 
[5] Goedheer, J.C. and Verhulsdonk, C.A.H. (1970) Fluorescence and phototransformation of protochlorophyll with etiolated bean leaves from $-196^{\circ} \mathrm{C}$ to $+20^{\circ} \mathrm{C}$. Biochemical and Biophysical Research Communications, 39, 260-266. doi:10.1016/0006-291X(70)90787-4

[6] Sironval, C. and Kuyper, P. (1972) The reduction of protochlorophyllide into chlorophyllide: IV. The nature of the intermediate $\mathrm{P}_{688-676}$ species. Photosynthetica, 6, 254-275.

[7] Raskin, V.I. (1976) Mechanism of photoredution of protochlorophyllide in the intact etiolated leaves. Vesti Akademii Nauk BSSR, 5, 43-46.

[8] Franck, F. and Mathis, P. (1980) A short-lived intermediate in the photoenzimatic reduction of protochlorophyll (ide) into chlorophyll(ide) at a physiological temperature. Photochemistry and Photobiology, 32, 799-803. doi:10.1111/j.1751-1097.1980.tb04058.x

[9] Inoue, Y., Kobayashi, T., Ogawa, T. and Shibata, K. (1981) A short lived intermediate in the photoconversion of protochlorophyllide to chlorophyllide $a$. Plant Cell Physiology, 22(2), 197-204.

[10] Dujardin, E. and Sironval, C. (1977) The primary reactions in the protochlorophyll(ide) photoreduction. Plant Science Letters, 10(1), 347- 353. doi:10.1016/0304-4211(77)90060-8

[11] Dujardin, E. and Correia, M. (1979) Long-wavelength absorbing pigment protein complexes as fluorescence quenchers in etiolated leaves illuminated in liquid nitrogen. Photobiochemistry and Photobiophysics, 1, 25-32.

[12] Dujardin, E., Correia, M. and Sironval, C. (1981) Fluorescence quenching of protochlorophyllide, chlorophyllide and chlorophyll in etiolated, greening and green leaves. Proceedings $5^{\text {th }}$ Internatioal Congress On Photosynthesis, Phyladelfia, 5, 21-29.

[13] Dujardin, E. (1984) The long-wavelength-absorbing quenchers formed during illumination of protochlorophyllide-proteins. In: Sironval, C. and Brouers, M. Eds., Protochlorophyllide Reduction and Greening, Martinus Nijhoff/Dr. W Junk Publisher, The Hague, 87-98.

[14] Losev, A.P. and Lyal'kova, N.D. (1979) Investigation of the primaries stages of protochlorophyllide photoreduction in the etiolated plants. Molecular Biology, 13, 837-844.

[15] Belyaeva, O.B. and Litvin, F.F. (1981) Primary reactions of protochlorophyllide into chlorophyllide phototransformation at $77 \mathrm{~K}$. Photosynthetica, 15, 210-215.

[16] Litvin, F.F., Ignatov, N.V. and Belyaeva, O.B. (1981) Photoreversibility of transformation of protochlorophyllide into chlorophyllide. Photobiochemistry and Photobiophysics, 2, 233-237.

[17] Heyes, D.J., Ruban, A.V., Wilks, H.M. and Hunter, C.N. (2002) Enzimology below $200 \mathrm{~K}$ : The kinetics and thermodinamics of the photochemistry catalyzed by protochlorophyllide oxidoreductase. Proceedings of the $\mathrm{Na}$ tional Academy of the Sciences of the USA, 99, 1114511150 . doi:10.1073/pnas.182274199

[18] Heyes, D.J., Ruban, A.V. and Hunter, C.N. (2003) Protochlorophyllide oxidoreductase: "Dark" reaction of a light-driven enzyme. Biochemistry, 42(2), 523-528. doi:10.1021/bi0268448

[19] Heyes, D.J., Hunter, C.N., van Stokkum, I.H.M., Grondelle, R. and Groot, M.L. (2003) Ultrafast enzymatic reaction dynamics in protochlorophyllide oxidoreductase.
Nature Structural Biology, 10, 491-492. doi:10.1038/nsb929

[20] Belyaeva, O.B., Personova, E.R. and Litvin, F.F. (1983) Photochemical reaction of chlorophyll biosynthesis at 4.2 K. Photosynthesis Research, 4(1), 81-85. doi:10.1007/BF00041803

[21] Belyaeva, O.B., Timofeev K.N. and Litvin, F.F. (1988) The primary reaction in the protochlorophyll(ide) photoreduction as investigated by optical and ESR-spectroscopy. Photosynthesis Research, 15(3), 247-256. doi:10.1007/BF00047356

[22] Belyaeva, O.B. (2009) Light dependent chlorophyll biosynthesis. BINOM, Moscow.

[23] Iwai, J., Ikeuchi, M., Inoue, Y. and Kobayashi, T. (1984) Early processes of protochlorophyllide photoreduction as measured by nanosecond and picosecond spectrophotometry. In: Sironval, C. and Brouers, M. Eds., Protochlorophyllide Reduction and Greening, Martinus Nijhoff/Dr.W Junk Publisher, The Hague, 99-112.

[24] Ignatov, N.V., Belyaeva, O.B. and Litvin, F.F. (1993) Low temperature phototransformation of protochlorophyll(ide) in etiolated leaves. Photosynthesis Research, 38(2), 117-124. doi:10.1007/BF00146410

[25] Frank, F., Dujardin E. and Sironval, C. (1980) Nonfluorescent, short-lived intermediate in photoenzymatic protochlorophyllide reduction at room temperature. Plant Sciences Letters, 18, 375-380. doi:10.1016/0304-4211(80)90101-7

[26] van Bochove, A.C., Griffiths, W.T. and van Grondelle, R. (1984) The primary reaction in the photoreduction of protochlorophyllide. A nanosecond fluorescence study. In: Sironval, C. and Brouers, M. Eds., Protochlorophyllide Reduction and Greening, Martinus Nijhoff/Dr.W Junk Publisher, The Hague, 113-125.

[27] Belyaeva, O.B. and Litvin, F.F. (1989) Photobiosynthesis of chlorophyll. Moscow State University Press, Moscow.

[28] Raskin, V.I. and Schwartz, A. (2002) The charge-transfer complex between protochlorophyllide and NADPH: An intermediate in protochlorophyllide photoreduction. Phosinthesis Research, 74(2), 181-186.

[29] Dobek, A., Dujardin, E., Franck, F., Sironval, C., Breton, J. and Roux, E. (1981) The first events of protochlorophyll(ide) photoreduction investigated in etiolated leaves by means of the fluorescence excited by short, $610 \mathrm{~nm}$ laser flashes at room temperature. Photobiochemistry and Photobiophysics, 2, 35-44.

[30] Schoefs, B., Bertrand, M. and Franck, F. (2000) Spectroscopic properties of protochlorophyllide analyzed in situ in the course of etiolation and in illuminated leaves. Photochemistry and Photobiology, 72(1), 85-93. doi:10.1562/0031-8655(2000)072<0085:SPOPAI $>2.0$.C $\underline{\mathrm{O} ; 2}$

[31] Ignatov, N.V. and Litvin, F.F. (2002) A new pathway of chlorophyll biosynthesis from long-wavelength protochlorophyllide Pchlide 686/676 in juvenile etiolated plants. Photosynthesis Research, 71(1), 195-207. doi:10.1023/A:1015595426181

[32] Belyaeva, O.B. and Sundqvist, C. (1998) Comparative investigation of the appearance of primary chlorophyllide forms in etiolated leaves, prolamellar bodies and prothylakoids. Photosynthesis Research, 55(1), 41-48. 
doi:10.1023/A:1005974305351

[33] Belyaeva, O.B. and Litvin, F.F. (2009) Pathways of formation of pigment forms at the terminal photobiochemical stage of chlorophyll biosynthesis. Biochemistry, 74, 1535-1544.

[34] Belyaeva, O.B., Bystrova, M.I., Safronova, I.A., Litvin, F.F. and Krasnovsky, A.A. (1985) Photoinduced reversible changes in protochlorophyll fluorescence in model systems. Molecular Biology, 30, 933-938.

[35] Brouers, M. (1975) Optical properties of in vivo aggregates of protochlorophyllide in non-polar solvents. II. Fluorescence polarization, delayed fluorescence and circular dichroism spectra. Photosynthetica, 9, 304-310.

[36] Böddi, B., Soos, J. and Lang, F. (1980) Protochlorophyll forms with different molecular arrangements. Biochimica et Biophysica Acta, 593(1), 158-165. doi:10.1016/0005-2728(80)90017-1

[37] Belyaeva, O.B., Griffiths, W.T., Kovalev, J.V., Timofeev, K.N. and Litvin, F.F. (2001) Participation of free radicals in photoreduction of protochlorophyllide to chlorophyllide in artificial pigment-protein complexes. Biochemistry, 66(2), 173-177.

[38] Heyes, D.J., Heathcote, P., Rigby, S.E.J., Palacios, M.A., Grondelle, R. and Hunter, C.N. (2006) The first catalytic step of the light-driven enzyme protochlorophyllide oxidoreductase proceeds via a charge transfer complex. The Journal of Biological Chemistry, 281(37), 26847-26853. doi:10.1074/jbc.M602943200

[39] Valera, V., Fung, M., Wessler, A. N. and Richards, W.R. (1987) Synthesis of 4R- and 4S-tritium labeled NADPH for the determination of the coenzyme sterespecificity of NADPH: Protochlorophyllide oxidoreductase. Biochemical and Biophysical Research Communications, 148(1), 515520. doi:10.1016/0006-291X(87)91141-7

[40] Begley, J.R. and Young, M. (1989) Protochlorophyllide reductase. I. Determination of the regiochemistry and the stereochemistry of the reduction of protochlorophyllide to chlorophyllide. Journal of the American Chemical Society, 111, 3095-3096. doi:10.1021/ja00190a071

[41] Lebedev, N., Karginova, O., McIvor, W. and Timko, M. (2001) Tyr275 and Lys279 stabilize NADPH within the catalytic site of NADPH: Protochlorophyllide oxidoreductase and are involved in the formation of the enzyme photoactive state. Biochemistry, 40(42), 12562-12574. doi:10.1021/bi0105025

[42] Menon, B.R.K., Waltho, J.P., Scrutton, N.S. and Heyes, D.J. (2009) Cryogenic and laser photoexitation studies identify multiple roles for active site residues in the light-driven enzyme protochlorophyllide oxidoreductase.
Journal of Biologycal Chemistry, 284(27), 18160-18166. doi:10.1074/jbc.M109.020719

[43] Townley, H.E., Sessions, R.B., Clarke, A.R., Dafforn, T.R. and Griffiths, W.T. (2001) Protochlorophyllide oxidoreductase: A homology model examined by sitedirected mutagenesis. Proteins: Structure, Funktion, and Genetics, Vol. 44(3), 329-335.

doi: $10.1002 /$ prot. 1098

[44] Menon, B.R.K., Davison, P.A., Hunter, C.N., Scrutton N.S. and Heyes, D.J. (2010) Mutagenesis alters the catalytic mechanism of the light-driven enzyme protochlorophyllide oxidoreductase. Journal of Biologycal Chemistry, 285(3), 2113-2119. doi:10.1074/jbc.M109.071522

[45] Krasnovsky, A.A. Jr., Belyaeva, O.B., Kovalev, Yu.V., Ignatov, N.V. and Litvin, F.F. (1999) Phosphorescence of intermediates of the terminal photochemical stage of chlorophyll biosynthesis. Biochemistry, 64(5), 703-708.

[46] Griffiths, W.T., McHugh, T. and Blankenship, R.E. (1996) The light intensity dependence of protochlorophyllide photoreduction and its significance to the catalytic mechanism of protochlorophyllide reductase. FEBS Letters, 398, 235-238. doi:10.1016/S0014-5793(96)01249-5

[47] Heyes, D.J., Sakuma, M., Visser, S.P. and Scrutton, N.S. (2009) Nuclear quantum tunneling in the light-activated enzyme protochlorophyllide oxidoreductase. Journal of biologibal chemistry, 284(6), 3762-3767.

[48] Belyaeva, O.B. and Litvin, F.F. (2007) Photoactive pigment-enzyme complexes of chlorophyll precursor. Biochemistry, 72(13), 1458-1477.

[49] Walker, C.J. and Griffiths, W.T. (1988) Protochlorophyllide reductase: A flavoprotein? FEBS Letters, 239(2), 259-262. doi:10.1016/0014-5793(88)80929-3

[50] Nayar, P., Brun, A., Harriman, A., Begley, T.P. (1992) Mechanistic studies on protochlorophyllide reductase: A model system for the enzymatic reaction. Journal of the Chemical Society, Chemical Communications, 5, 395-397. doi:10.1039/c39920000395

[51] Ignatov, N.V., Belyaeva, O.B. and Litvin, F.F. (1993) The possible role of the flavin components of protochlorophylide-protein complexes in the primary processes of protochlorophyll photoreduction in etiolated plant leaves. Photosynthetica, 29, 235-241.

[52] Blenkenhorn, G. (1976) Nicotinamide-dependent one-electron and two-electron (Flavin) oxidoreductation: thermodynamics, kinetics, and mechanism. European Journal of Biochemistry, 67(1), 67-80. doi:10.1111/j.1432-1033.1976.tb10634.x 